\title{
The changing scope of difficult airway management
}

\author{
Jonathan P. Wanderer, MD · Jesse M. Ehrenfeld, MD • \\ Warren S. Sandberg, MD, PhD • Richard H. Epstein, MD
}

Received: 22 May 2013/Accepted: 10 July 2013/Published online: 30 July 2013

(C) Canadian Anesthesiologists' Society 2013

\section{To the Editor,}

Over the past decade, the introduction of video laryngoscopes has resulted in dramatic changes in how we secure difficult airways. Consequently, anesthesia societies worldwide are including these devices in their clinical and training guidelines (e.g., Association of Canadian University Departments of Anesthesia [ACUDA] 2010 National Curriculum for Canadian Anesthesia Residency, ${ }^{\mathrm{A}}$ the 2012 Australian and New Zealand Guidelines on Equipment to Manage a Difficult Airway During Anaesthesia, ${ }^{\mathrm{B}}$ and the 2013 American Society of Anesthesiologists [ASA] Practice Guidelines for Management of the Difficult Airway). ${ }^{1}$ Over the same time, advances in supraglottic airways (SGAs) have given practitioners additional options. While awake fibreoptic intubation (FOI) is still considered the mainstay of known difficult airway management, recent work has shown that awake video laryngoscopy and FOI may provide similar results. $^{2}$ These technological advances (e.g., SGA and

J. P. Wanderer, MD (凶) · J. M. Ehrenfeld, MD .

W. S. Sandberg, MD, PhD

Department of Anesthesiology, Vanderbilt University, Nashville, TN, USA

e-mail: Jonathan.p.wanderer@vanderbilt.edu

J. M. Ehrenfeld, MD - W. S. Sandberg, MD, PhD

Department of Biomedical Informatics, Vanderbilt University, Nashville, TN, USA

J. M. Ehrenfeld, MD

Department of Surgery, Vanderbilt University, Nashville, TN, USA

R. H. Epstein, MD

Department of Anesthesiology, Jefferson Medical College, Philadelphia, PA, USA video laryngoscopy), in conjunction with guideline changes, have substantially altered the practice of general anesthesia.

We report a single-institution analysis of 12 years of anesthetic records that shows a rise in the use of supraglottic airways, Eschmann ${ }^{\circledR}$ Tracheal Tube Introducers (bougies), and video laryngoscopes while observing a decrease in difficult airways. This work was approved by the Institutional Review Board at Vanderbilt University (protocol 110613). Our institution utilizes the GasChart application which was developed in-house as our anesthesia informatics management system and is commercially available. ${ }^{\mathrm{C}}$ Airway management within our system is documented in a structured manner where usage of a SGA, fibreoptic scope, bougie, and video laryngoscope is charted discretely. Additionally, difficulty of intubation is documented as easy, moderate, difficult, or impossible by the anesthesia provider. For this analysis, difficult intubations were defined as those documented as difficult or impossible.

We identified 542,993 anesthetics in adults from March 2001 through February 2013 and analyzed airway management by quarter in the 317,509 records where placement of an endotracheal tube or SGA was documented. We created an infographic ${ }^{3}$ to examine

\footnotetext{
A National Curriculum for Canadian Anesthesia Residency. Available from URL: http://anesthesiology.queensu.ca/assets/National Curriculum_final_v2.pdf (accessed May 28th, 2013).

B Guidelines on Equipment to Manage a Difficult Airway During Anaesthesia. Available from URL: http://www.anzca.edu.au/resources/ professional-documents/documents/professional-standards/pdf-files/ PS56-guidelines-on-equipment-to-manage-a-difficult-airway-duringanaesthesia.pdf (accessed May 28th, 2013).

C Acuitec GasChart. Available from URL: http://gaschart.acuitec. com/about/ (accessed May 28th, 2013).
} 


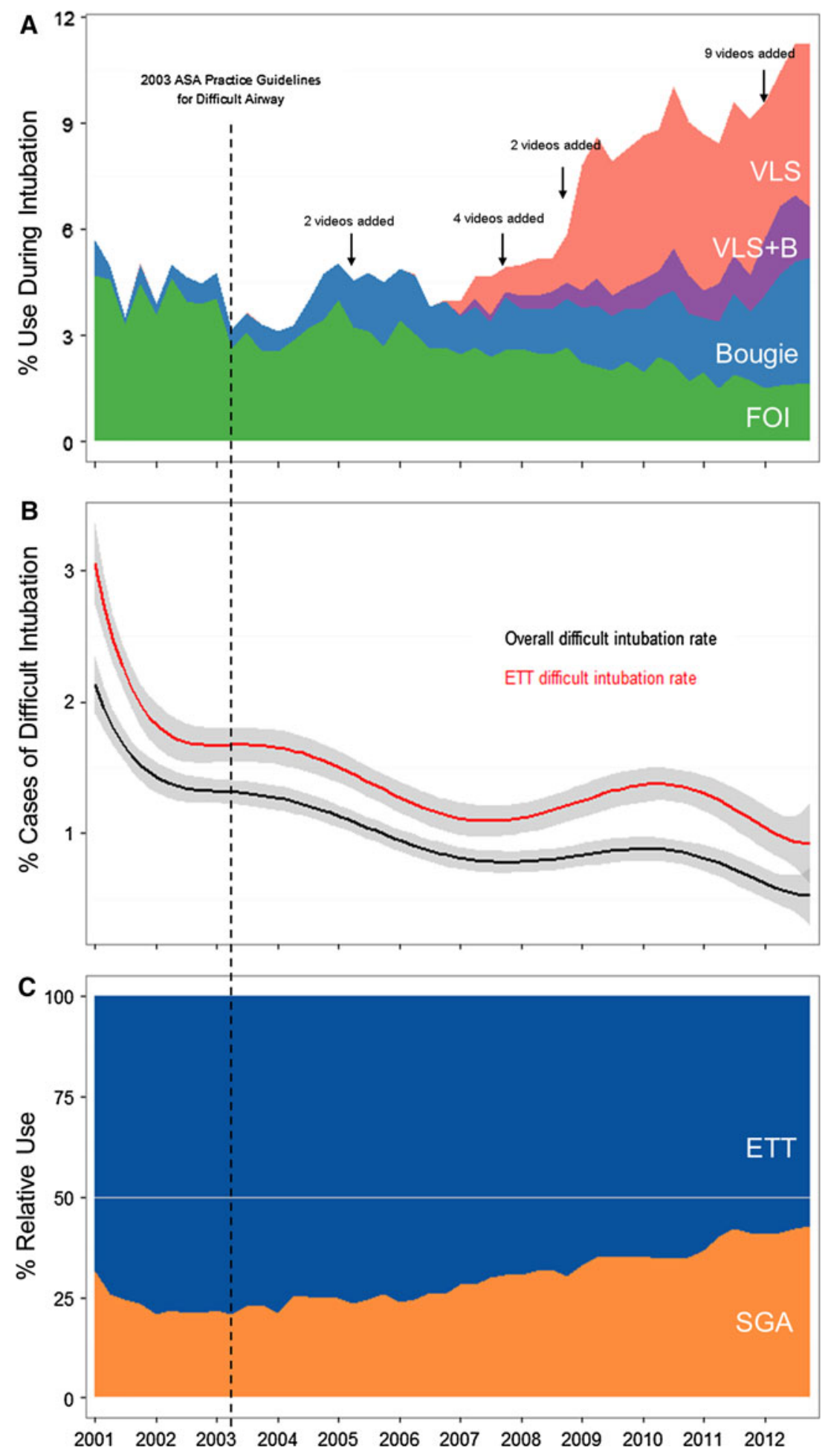

Figure Airway management infographic: A) Percentage of all cases utilizing fibreoptic intubation (FOI), bougie-assisted direct laryngoscopy (Bougie), video laryngoscopy alone (VLS), and bougieassisted video laryngoscopy (VLS+B). B) Percentage of cases with difficult intubation as indicated by the overall rate (fraction of endotracheal tube [ETT] + supraglottic airway [SGA], black line) and the rate within cases with ETT placement (fraction of ETT only, red line), plotted as LOcal regrESSion (LOESS) lines (95\% confidence interval, gray area). During the first quarter of analysis, 45 difficult intubations were identified among 1,549 ETT and 721 SGA placements. During the last quarter, 59 difficult intubations were identified among 6,528 ETT and 4,903 SGA placements. C) Relative usage of ETTs and SGAs 
trends in airway management at our institution that overlap with the publication of the 2003 ASA Difficult Airway Practice Guidelines (dashed black line). ${ }^{4}$ Acquisition of video laryngoscopes (arrows, Figure A) was associated with increased use of video laryngoscopes (pink) and decreased use of FOI (green). Use of bougies rose, both during direct laryngoscopy (blue) and in conjunction with video laryngoscopy (purple). Interestingly, there was a decline in the percentage of endotracheal intubations subjectively deemed difficult as documented by anesthesia providers (red line, Figure B). During this time, the usage of SGAs rose steadily (Figure C). The decline in difficult endotrachial intubations was also recognized by the number of difficult intubations proportionate to the number of airways managed with either or both techniques (black line, Figure B).

While there is no universal definition of the difficult airway, ${ }^{5}$ and these observations do not prove causation, our data indicate that there has been a substantial change in how we manage the airway and that this change may be related to technological development. As these devices were introduced into our institution, clinical training opportunities were provided to our anesthesia providers. Even so, no institutional encouragement was given to utilize any specific airway management modalities. Together, these data suggest that advances in airway technology may have changed management over time.
Funding source Departmental funding.

Competing interests None of the authors have any financial interest in any corporations involved in the development or marketing of the devices described in this report.

\section{References}

1. Apfelbaum JL, Hagberg CA, Caplan RA, et al. Practice guidelines for management of the difficult airway: an updated report by the American Society of Anesthesiologists Task Force on Management of the Difficult Airway. Anesthesiology 2013; 118: 251-70.

2. Rosenstock CV, Thogersen B, Afshari A, Christensen AL, Eriksen $C$, Gatke MR. Awake fiberoptic or awake video laryngoscopic tracheal intubation in patients with anticipated difficult airway management: a randomized clinical trial. Anesthesiology 2012; 116: 1210-6.

3. Wanderer JP, Ehrenfeld JM, Sandberg WS, Rathmell JP. Infographics in anesthesiology: a volatile tale of two institutions. Anesthesiology 2013; 118: 1456.

4. American Society of Anesthesiologists Task Force on Management of the Difficult Airway. Practice guidelines for management of the difficult airway: an updated report by the American Society of Anesthesiologists Task Force on Management of the Difficult Airway. Anesthesiology 2003; 98: 1269-77.

5. Souvatzis X, Askitopoulou H. Definition of difficult tracheal intubation. Eur J Anaesthesiol 2008; 25: 694-5. 